\title{
MI-6: Michigan interferometry with 6 telescopes
}

\author{
John D. Monnier ${ }^{a}$, M. Anderson ${ }^{a}$, F. Baron ${ }^{a}$, D. H. Berger ${ }^{a}$, X. Che ${ }^{a}$, T. Eckhause $^{a}$, S. Kraus ${ }^{a}$ \\ , E. Pedretti ${ }^{b}$, N. Thureau ${ }^{b}$, R. Millan-Gabet ${ }^{c}$, T. ten Brummelaar ${ }^{d}$, P. Irwin ${ }^{e}$, M. Zhao $^{f}$ \\ ${ }^{a}$ Department of Astronomy, University of Michigan, 500 Church Street, Ann Arbor, MI 48109; \\ ${ }^{b}$ University of St. Andrews, Scotland, UK; \\ ${ }^{c}$ Caltech, Pasadena, CA; \\ ${ }^{d}$ CHARA, Georgia State University, Atlanta, GA; \\ ${ }^{e}$ Sierra Madre, CA; \\ fJet Propulsion Laboratory, Pasadena, CA
}

\begin{abstract}
Based on the success of four-telescope imaging with the Michigan Infrared Combiner (MIRC) on the CHARA Array, our Michigan-based group will now upgrade our system to combine all six CHARA telescope simultaneously. In order to make this observationally efficient, we have had to improve a number of subsystems and commission new ones, including the new CHAMP fringe tracker, the introduction of photometric channels, the upgrading of the realtime operating systems, and the obvious hardware and software upgrades of the control system and the data pipeline. Here we will discuss the advantages of six-telescope operation, outline our upgrade plans and discuss our current progress.
\end{abstract}

Keywords: interferometry, infrared

\section{INTRODUCTION}

True interferometric imaging at visible and infrared wavelengths is practically impossible with only 2 telescopes due to the corruption of phase information from atmospheric turbulence. Three telescopes allow one closure phase to be measured at a time which salvages imaging capabilities but requires excessively long observing campaigns to reconstruct images more complicated than a binary or triple system.

The University of Michigan group led by John Monnier has focused on demonstrating high-quality aperturesynthesis imaging using today's long-baseline interferometric arrays. The Michigan Infrared Combiner (MIRC) $)^{1,2}$ was the first combiner truly optimized for imaging and was originally commissioned in 2006 on the CHARA ${ }^{3}$ Array, a six-telescope interferometer on Mt. Wilson built and operated by Georgia State University (PI: Hal McAlister). MIRC still stands as the only operational combiner for 4 (or more) telescopes that measures all fringe visibilities and closure phases at the same time (soon to be joined by combiner PIONIER on VLTI; see Berger et al. these proceedings). The number of beams a combiner can interfere is crucial to the efficiency of operation: a 4-beam combiner will measure 6 visibilities/4 closure phases simultaneously, compared with 3 visibilities/1 closure phase for a 3-telescope array. On a practical level, this factor of 3 increase in speed has made a huge difference in scientific productivity.

While there are some sensitivity disadvantages to the MIRC design, we note that the most "interesting" objects amenable to aperture synthesis imaging are not faint. In order for imaging to be useful on a complicated object, the characteristic size scale must be many angular resolution elements across. For broadband imaging of stars, this "size" requirement assures us that suitable imaging targets are always "bright" by even interferometric standards (indeed, many classic, prototype objects are no longer observable by modern arrays because they are too big, e.g., Betelgeuse). While it is true that imaging thermal emission from circumstellar dust (in mass-losing stars or young stars) or imaging at very high spectral resolutions is indeed still challenging, these science objects are approachable by using a dedicated broadband fringe tracker associated with a particular science instrument.

Further author information: (Send correspondence to J.D.M.)

J.D.M.: E-mail: monnier@umich.edu, Telephone: 17347635822

Optical and Infrared Interferometry II, edited by William C. Danchi, Françoise Delplancke, Jayadev K. Rajagopal, Proc. of SPIE Vol. $7734,77340 \mathrm{G} \cdot$ ( ) 2010 SPIE · CCC code: 0277-786X/10/\$18 · doi: 10.1117/12.858286 

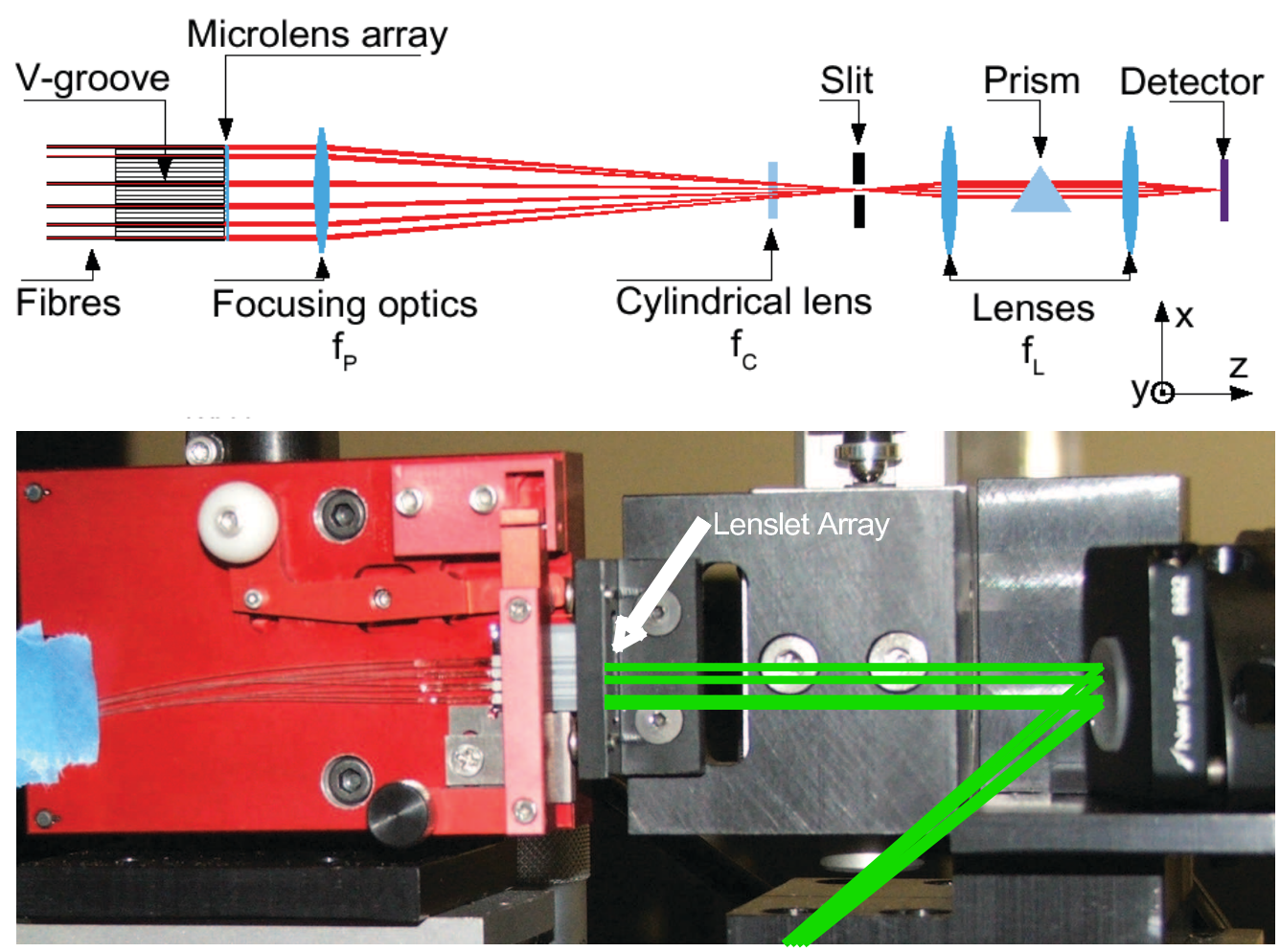

Figure 1. The overall schematic of the MIRC instrument can be seen in the top part of diagram. Once light from each telescope is focused into a single mode fiber, the fibers are arranged into a non-redundant pattern. The bottom image shows the fibers packaged in a silicon v-groove array, the location of the collimator lenslet array, and a spherical silver mirror that focuses the beams to produce interference fringes at the input slit of the camera-spectrometer. The newlycommissioned photometric channels" (discussed in Section 3.2) pick-off the beams just after the lenslet array and before the spherical mirror pictured here.

In a world with kilometric baseline interferometers, sensitivity will again become an issue for imaging more distant stars but no array currently under construction has baselines more than $350 \mathrm{~m}$, unfortunately (excepting OHANA).

\section{LESSONS FROM MIRC IMAGING AT CHARA}

\subsection{Brief Description of MIRC}

The MIRC combiner was first described at the 2004 SPIE meeting by Monnier et al. ${ }^{1}$ and a description of commissioning and first scientific results can be found in the 2006 and 2008 SPIE proceedings. ${ }^{2,4}$

MIRC is an image-plane combiner, allowing simultaneous measurements of all available baselines and closure phases in the full 6-telescope CHARA array (although currently we only combine 4 telescopes at a time). The beams from all telescopes are arranged in a linear non-redundant pattern and then imaged onto the slit of an infrared spectrograph at the backend. In order to allow precision calibration of visibility amplitudes and closure phases, the synthetic pupil is formed using light transported and spatially-filtered by single-mode fibers for optical stability. ${ }^{5}$ A non-deviating prism pair $(\mathrm{R} \sim 42)$ or low-resolution grism $(\mathrm{R} \sim 150,450)$ on the output mitigates dispersion problems and bandwidth-smearing of data (especially important with the long CHARA baselines).

Here we only briefly summarize the main properties of the MIRC combiner:

- Image Plane Combination: 6 telescopes, 15 visibilities, 20 closure phases simultaneously; to date, we have used only 4 telescopes ( 6 visibilities, 4 closure phases simultaneously) 


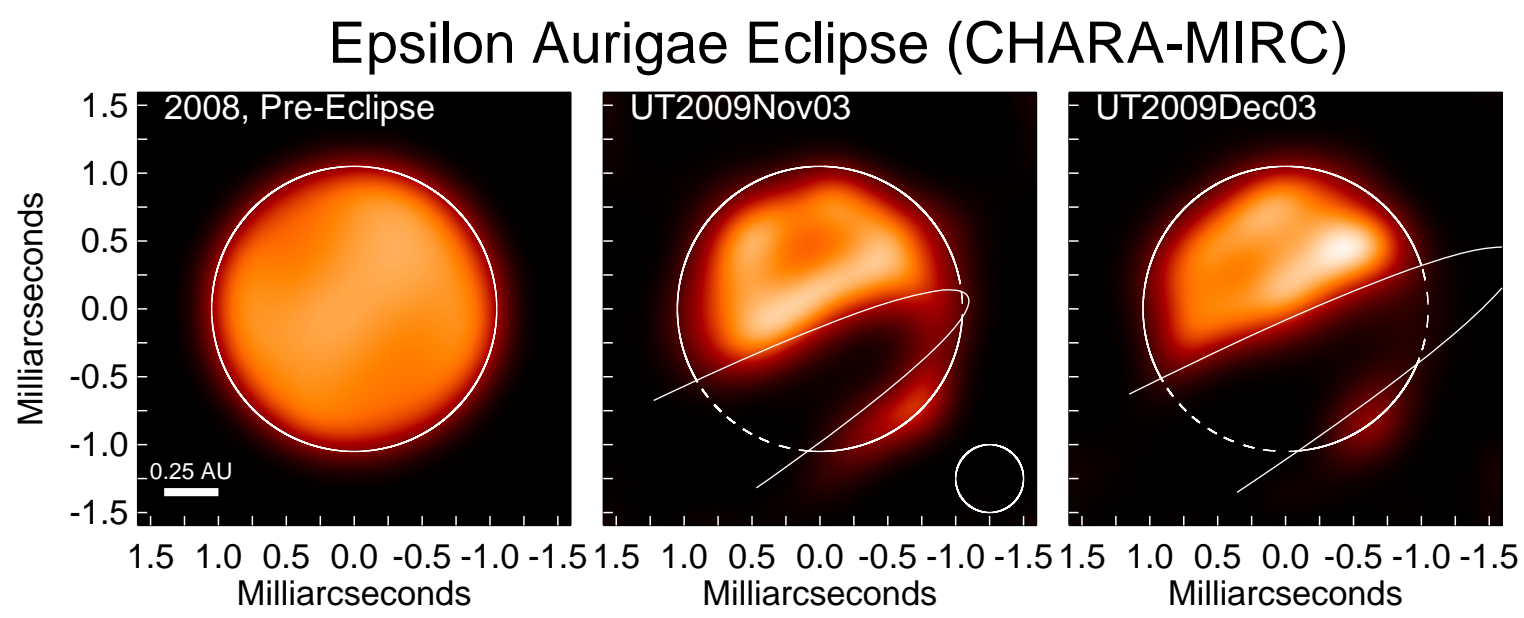

Figure 2. Kloppenborg ${ }^{10}$ et al (2010) used MIRC to the transiting disk in the epsilon Aurigae system. Each image was made based one data from typically two consecutive observing nights using MACIM ${ }^{11}$ algorithm.

- spectral range 1.45-2.5 microns (H or K bands)

- Low-resolution spectroscopy, $\mathrm{R} \sim 42,150$ or 450

- Spatial filtering using single-mode fibers

- Integration with a separate fringe tracker (CHAMP, see Berger et al. $\left.{ }^{6}\right)$.

\subsection{Imaging Successes}

We want to point to the diversity of imaging results using MIRC at CHARA as a validation of the design philosophy:

1. Rapid Rotators. We have made ground-breaking images of main sequence stars besides the Sun. We have focusing on rapid rotators because they have non-trivial and interesting morphologies, and our results impact the important field of massive star evolution. We have created aperture synthesis images of Altair, ${ }^{7}$ Alderamin and Rasahague, ${ }^{8}$ Regulus and $\beta$ Cas (Che et al, in preparation).

2. Interacting Binaries. We have made the first images of interating binary stars, so far a published "movie" of $\beta$ Lyrae, ${ }^{9}$ and new work on the triple system Algol (Baron et al. in preparation).

3. $\epsilon$ Aurigae. This fascinating system gave us the once-in-a-generation opportunity to image its large opaque transiting disk ${ }^{10}$ as shown in Figure 2. These rather complex images required a novel two-configuration observing strategy designed by Gail Schaefer (CHARA, GSU) whereby we used all 6 telescopes each night, starting with S1-E1-W1-W2 and then switching S2-E2-W1-W2, with no need to do major realignments during the night. This method gave us 8 of the possible 20 closur ephases and 11 of the 15 possible baselines in a single night with the existing 4-beam MIRC.

4. Disks around Be stars. We are monitoring the time-variable disk around Be stars - these objects are hard to image due to their relatively high visibilities (e.g., Schaefer et al., submitted).

5. Spotted Stars. We are currently working on two projects to image the rotating spots on magnetically active RS CVN stars (Pedretti et al., Parks et al.; both in preparation). We have found the great complexity of the spot patterns observed to be difficult to unambiguously reconstruct with only 4 telescopes - another motivation to move to 6 telescopes to increase the uv coverage. 
Actual UV Coverage for Regulus

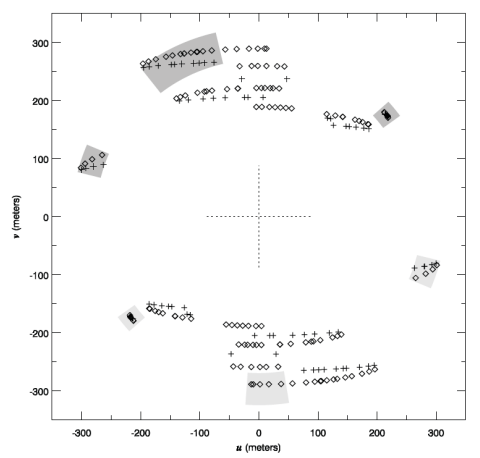

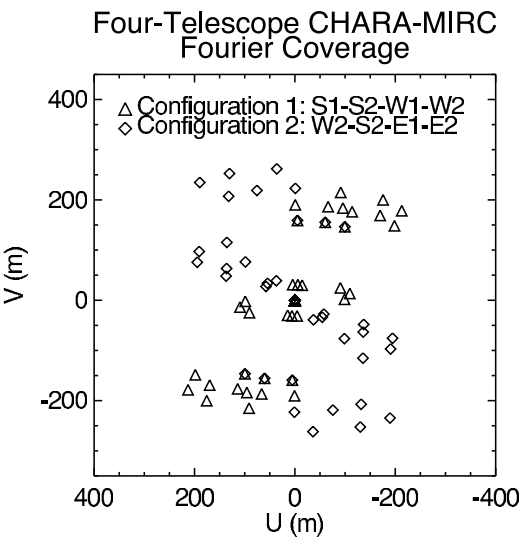

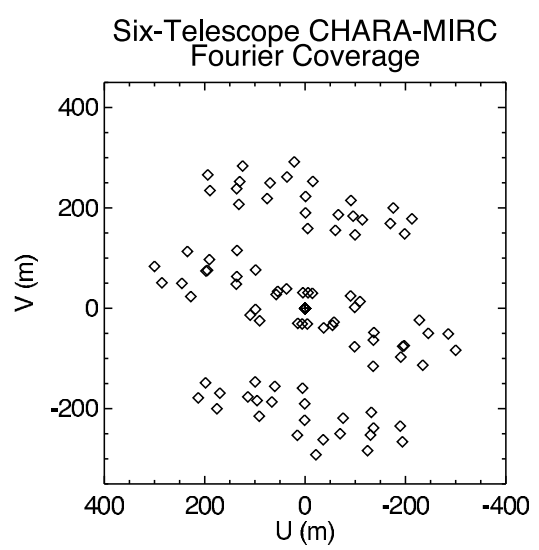

Figure 3. The left panel shows the actual UV-coverage from 22 observing nights at CHARA for Regulus ${ }^{19}$ when limited to using a single-baseline (2-telescopes) combiner. An equivalent Fourier coverage can be obtained in two nights with the current MIRC combiner using 4 telescopes (middle panel). MIRC will soon be upgraded to use all 6 telescopes and the right panel shows the UV-coverage attainable in a single night.

These breakthroughs would not have been possible without advances in interferometric imaging software. Following the recent standardization of optical interferometry FITS format (OI-FITS ${ }^{12}$ ), a number of useful imaging packages have emerged that can use the OI-FITS standard. Our group has found the BSMEM ${ }^{13,14}$ and MACIM $^{11}$ packages the most practical to use with our data, although other interesting packages that can be found in past image "Beauty Contest" papers. ${ }^{15-17}$ There are also additonal papers on this topic in these new SPIE proceedings (e.g., Baron et al.).

While most of the motivation for the 6-beam MIRC upgrade is based on enhancing imaging capabilities, there is also an exoplanet science case that takes advantage of the closure phase precision attainable using single-mode fibers. For more information on this application, please see the previous SPIE paper ${ }^{18}$ and the new paper by Zhao et al. in these proceedings.

\subsection{Advantages}

The relative advantages and disadvantages of different combiners have been debated for decades in the pages of the SPIE proceedings. What is usually missing is any sense of practical implementation. While theoretical calculations of signal-to-noise ratio and imaging fidelity can be illuminating, a pragmatist will look to success in "real-world" implementation as well. Indeed, there is an understandable fetish surrounding optimizing combiners for sensitivity, but we hold that practical constraints are also crucial to "on-sky" astronomical success. In real life, observing time is limited, telescopes are hard to move around and re-align, and there can be cross-talk between fringes in all-in-one, temporally-modulated beam combiners.

Indeed, another real metric (in addition to limiting magnitude or sensitivity) is how many scientificallyuseful results can be attained per observing night. Here we will outline some practical advantages of all-in-one, spatially-modulated beam combiners such as MIRC:

- SPEED. A four-beam combiner collects visibilities at twice the rate and closure phases 4 times faster than a 3-beam system. This dramatic speed up means that only one or two nights of data are adequate to make an image. ${ }^{7}$ Also, many objects change on the time scale it takes to accumulate Fourier coverage, ${ }^{9}$ which makes imaging impossible for 3-beam combiners. Given these issues and the additional difficulties of obtaining sufficient observing time from stingy Time Allocation Committees and overcoming the inevitable downtime from weather and equipment failures, the practical advantage of SPEED is immensely important to increase the scientific publication rate and to draw more astronomers into our field. Indeed - the increasing competition for observing nights at CHARA has led to a reduction in available observing time for my group, and this scarcity has been a prime motivator for the 6-telescope upgrade to MIRC, following our success with 4 telescopes. For the proposed 6-telescope upgrade, we will get 15 visibilities and 20 
closure phases simultaneously, putting CHARA nearly on par with radio/mm-wave imaging arrays such as SMA, OVRO, BIMA, and VLBI.

- BOOTSTRAP options. For a three beam combiner, one must be able to track 2 of the 3 fringes or one can not measure a closure phase nor all the visibilities. For a four-beam combiner, one needs to only 3 of the 6 fringes in order to track*. Many closure phases are impossible to detect using a 3-beam combiner because 2 of the 3 fringes will be weak. However, for a $4+$ beam combiner there are many "cross" fringes that can help to phase up the array for tracking atmospheric fluctuations - this is a somewhat subtle point but leads to a much greater efficiency and ease in observation planning. For instance, at CHARA one can have 3 telescopes out at the edge of the array and one (pseudo-)central station - thus one can find 3 short baseline fringes for fringe tracking and get data on all triangles even for very faint long-baseline fringes. In "real life", it ranges from difficult to impossible to move and re-align telescope stations and so 4+ telescope arrays are essential to make good use of the array time without constant shuffling of telescope locations to deal with bootstrap issues. Also, an all-in-one combiner will naturally use all the bright fringes so one does not need to worry about source geometry in so much detail in observation planning. As a real-world example, at CHARA we can always track fringes with MIRC on stars we resolve even to the 2nd or 3rd lobe of the visibility pattern - not by clever timing to make sure the baselines sample the peaks of the "visiblity bounces," but rather just because there happens to 'always' be enough high visibility fringes in practice on some combination of baselines (having some low to moderate spectral resolution helps here to avoid bandwidth smearing for highly resolved objects). This latter benefit is especially true for complicated objects where it is difficult to predict the fringe visibilities ahead of time, such as for young stellar objects. ${ }^{20}$

Parenthetically - we note that a pair-wise combiner that captures all beam combinations will probably be superior to the image-plane combination scheme of MIRC (due to extra readnoise). Unfortunately, pair-wise combiners in bulk optics have a large footprint and are hard to maintain alignments when using a large number of beams. A promising strategy to implement a pairwise scheme employing ABCD modulation using integrated optics $^{21}$ could offer a robust and superior solution to MIRC (see paper on new VLTI combiner project PIONIER in these proceedings).

\subsection{Limitations}

As has been discussed in previous MIRC papers, the MIRC design philosophy sacrificed sensitivity in order to optimize uv-coverage and calibration precision. On a practical level, MIRC has had a number of other practical limitations that have kept it and its user base from making optimal use of the data.

- SENSITIVITY. MIRC combines light in an all-in-one fashion which is not quite as sensitive as a wellimplemented pairwise system. Also MIRC uses spatial filtering to allow good calibration and disperses the fringes over 8 channels $(\mathrm{R} \sim 42)$ to increase the coherent field-of-view and to minimize systematic errors due to unmatched air dispersion. The spectrograph and synthetic pupil are warm which means that K-band observations are marginal due to factor $>100$ times excess thermal background (a cryogenic MIRC would be a practical upgrade in the future). One could expect the gain of up to 4 magnitudes by doing pairwise beam combination using broadband and no fibers - and this is what we have done by building the CHAMP fringe tracker (see $\S 3.1$ ).

- CALIBRATION. The original MIRC did not have photometric "taps" to monitor the realtime coupling fluctuations from each telescope. Up until 2009, our system relied on somewhat indirect methods discussed in detail in a previous SPIE paper. ${ }^{4}$ We generally have found about 10-20\% systematic errors in our $V^{2}$ measurement which limit the precision of some science efforts, such as limb-darkening measurements and high-visibility science (such as imaging free-free emission around Be stars). We have instituted new "photometric channels" that appear to improve calibration by a factor of $\sim 3$ and this is discussed more in $\S 3.2$ and in the Che et al. contribution in these proceedings. Note that calibration of a pair-wise scheme would be more robust to coupling fluctuations since amplitude calibration is robust for 50:50 (two-beam) combiner even for large uncertainties in the flux contributions from the individual beams.

\footnotetext{
${ }^{*}$ Not any set of 3 fringes will do but most sets of three are ok - there must be a fringe with at least every telescope.
} 


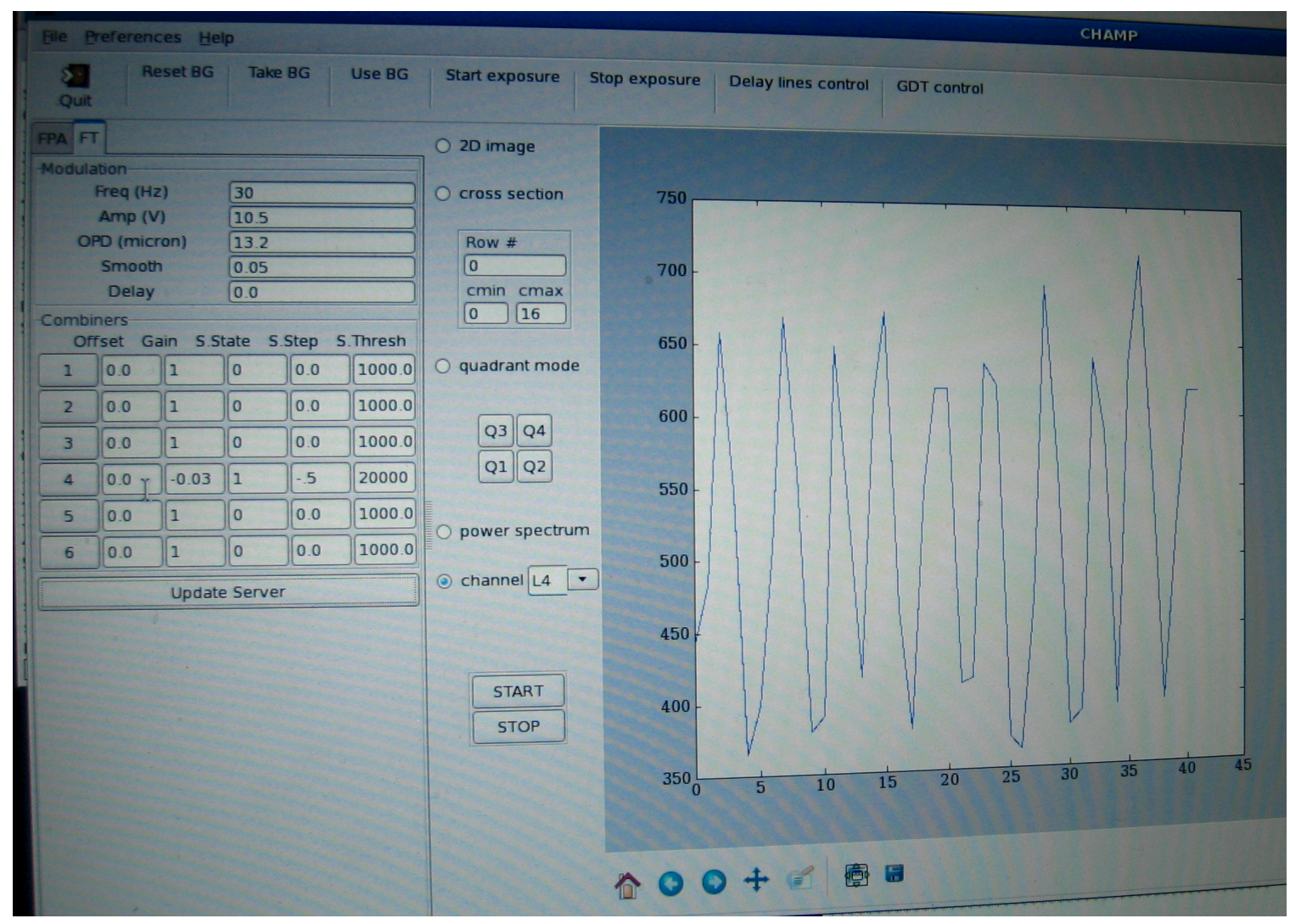

Figure 4. This is a photograph of first "fringe lock" of the CHARA fringe tracker CHAMP ${ }^{6}$ on the bright test star $\beta$ Cas on UT2009Aug19.

- OBSERVING INEFFICIENCIES. Currently we have to spend a few hours before each MIRC run using the CHARA CLASSIC combiner to "find fringes." This is because it takes many hours to search for fringes after a configuration change, since the fringe offsets can be up unknown up to a few $\mathrm{cm}$. The CHAMP fringe tracker should eliminate this step by searching more quickly and automatically. Also, we currently have to spend about $75 \%$ of the observing time taking "shutter" sequences in order to align each fiber and to calibrate the photometry from each telescope. With dedicated "photometric channels" we can drastically reduce the amount of time peaking up fibers since they can now be done in parallel. This also means we need far fewer frames of shutter data to calibrate our methods.

Upgrading MIRC to a 6-beam system alone would have exacerbated the difficulties described above. It is only because we are combining our 6-beam upgrade with both the photometric upgrade and the fringe tracker upgrade that we can expect to see dramatic improvements sensitivity, calibration, and observing efficiency.

\section{THE MIRC UPGRADE}

As discussed above, the key hardware upgrades needed for a successful six-telescope upgrade are fringe tracking and photometric channels for improved sensivity, calibration precision, and observing efficiency. In addition, this require some important computer and software upgrades as detailed below.

\subsection{Progress report: CHAMP fringetracker}

We discussed the progress of the CHAMP fringe tracker in $2008 .{ }^{6}$ Since that time, we shipped the entire instrument to Mt. Wilson in 2009 July. We installed the optics and executed a single-baseline commissioning run 

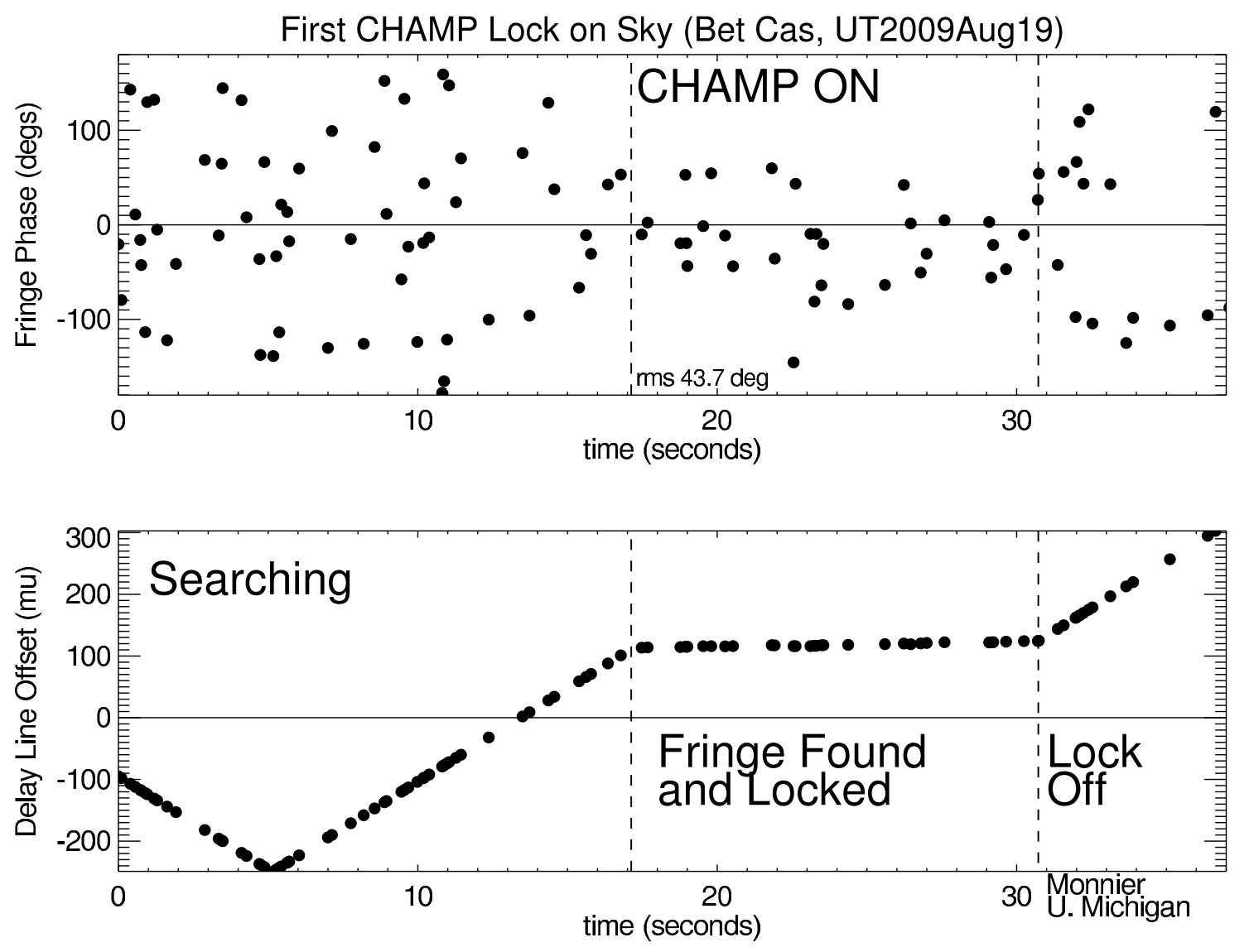

Figure 5. Left panel shows the position of the delay line during the inital fringe search, the fringe lock period and then following unlocking the fringe tracker. The right-hand panel shows an plot of the the fringe un-lock.

in early 2009August. We successfully locked on fringes on UT2009Aug19 using the star $\beta$ Cas and a screenshot of this technical milestone can be found in Figure 4 (video at http://www.youtube.com/watch?v=Co6pbuSYlTA)

At that time, we had only a crude way to save diagnostic data and a short (incomplete) snapshot of the fringe tracker performance can be seen in Figure 5. We see an rms phase error of about 0.75 radians when the fringe tracker was locked, which is not atypical compared to other similar fringe trackers. ${ }^{22}$ Note that we later determined the fringe tracker update rate was about $2 \times$ slower than we had expected due to a network buffering setting (nagle!) that limited the bandwidth of our fringe tracker servo loop; we have since eliminated this buffering and expect even better performance in the future.

\subsection{Progress report: MIRC photometric channels}

As discussed above, one of the long-standing limitations of the MIRC combiner has been the lack of realtime photometric taps to monitor the coupled flux from each telescope during fringe acquisition. We installed a prototype photometric channel system on-site in August 2009 and it was used throughout the Fall. We have actually stopped using the other methods of photometric measurement (i.e, the chopper). Many details of this new subsystem can be found in another paper in this proceedings (Che et al, these proceedings).

Here we will show one figure illustrating the data quality improvement we have seen with the new PC system. Figure 6 shows a calibrated visibility for a well-resolved star. The left panel shows the MIRC result using a chopper-based method for calibrating the telescope fluxes. The right panel shows the same fringe data but calibrated using the photometric channel flux measurements. The improvement in calibration was dramatic for this example. 

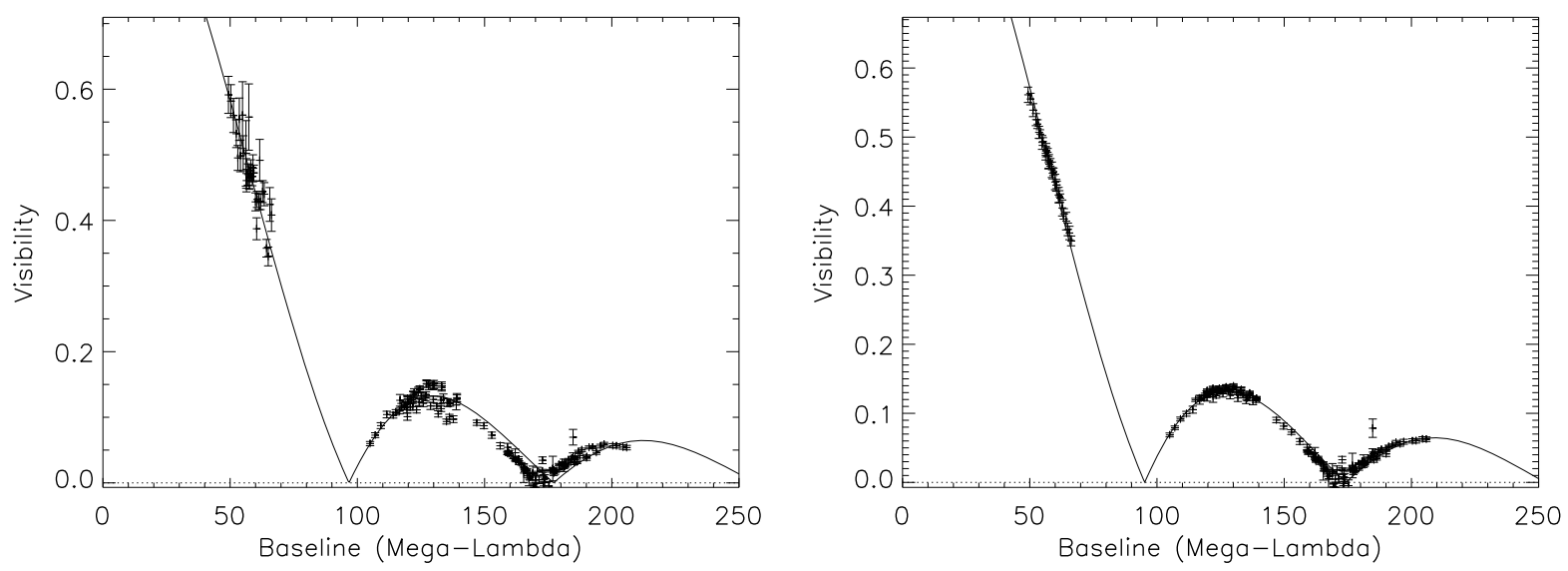

Figure 6. Engineering data using the photometric channels on MIRC for the first time. The left panel shows a calibrated visibility curve using the previous chopper-based method for calibration while the right hand panel shows results using the photometric channels. Please see paper by Che et al. in these proceedings for more details on this new mode.

\subsection{Progress report: Operating system upgrades}

\subsubsection{RTAI-fusion and Xenomai}

The Xenomai project aims to create a completely open-source real time operating system based on the Linux kernel. The project started in 2001. In 2003 Xenomai and the real-time application interface (RTAI) merged their effort to create the RTAI-fusion Linux-based real-time system. The new project inherited some of the advantages of Xenomai and RTAI, in particular the ability of running real-time processes in user space instead of kernel space and the inherited advantage of being able to use most of the Linux user-space libraries without modification. The new real-time system was also capable of using "skins" that enabled the use of programmes written for other real-time APIs, such as VxWorks, pSOS+, VRTX, ulTRON, RTAI with relatively little modification.

In 2005 Xenomai and RTAI split again because of disagreement on future direction of the OS among the major developers. RTAI-fusion became a dead-end but xenomai inherited its functionality. This split impacted the maintainability of the MIRC real-time system that was based on an experimental version of RTAI-fusion installed on a Gentoo Linux distribution. At the time the easiest way to install RTAI-fusion was using the portage system of Gentoo. Nowadays Xenomai is present as a Debian package in the Debian Linux distribution and is easily installable on that platform. The transition from RTAI-fusion to Xenomai is also relatively easy and well documented.

\subsubsection{Transition from MIRC to CHAMP}

MIRC and CHAMP were developed roughly 3 years apart. CHAMP was developed on Xenomai based on a Linux Gentoo distribution unlike MIRC that was based on RTAI-fusion. ${ }^{23}$ Ideally we would like to have MIRC and CHAMP based on the same Linux distribution, using the same Linux kernel and same version of Xenomai. The use of proprietary drivers made the transition difficult. An additional SATA disc controller on MIRC used a proprietary driver not well-supported in the Linux kernel. This was also the case for the Astrocam driver. CHAMP inherited the same problems with the addition of proprietary drivers for the National Instruments PCI-6711 card, used for driving the piezo scanners. To make the situation worse the manufacturer of the disc controller dropped the support for the SATA card, consequently CHAMP newer OS could not use the SATA card any more.

\subsubsection{The present situation}

The goal of the current effort is to use the same linux distribution (Debian stable) and kernel on both MIRC and CHAMP. The additional disc controller is now supported natively by the linux kernel so there is no more need for proprietary drivers. The astrocam driver is a source-code tar-ball and follows loosely the kernel development. We have managed to compile successfully this driver so far but there are some incompatibilites that need attnention. 
The proprietary drivers for the PCI-6711 card are supplied only for Mandrakelinux 10.1 Official, Mandriva Linux 2006, SUSE LINUX Professional 9.2 and 9.3 and Red Hat Enterprise Linux WS 3. Fortunately there are opensource drivers that support this card natively in Xenomai (Analogy drivers). The advantage of open-source drivers is that they track the OS distribution, they are continuously improved and they allow to upgrade the OS rendering the machine more maintainable.

Table 1. Current planned timeline for MIRC 6-telescope upgrade, with associated photometric channels and fringe tracker full on-sky commissioning and validation

\begin{tabular}{|l|l|}
\hline 2010 Summer & $\begin{array}{l}\text { Upgrade software interface for photometric channels } \\
\text { Upgrade MIRC and CHAMP to same xenomai OS } \\
\text { Finish reatlime and interface software for CHAMP }\end{array}$ \\
\hline 2010 Fall & $\begin{array}{l}\text { Full commissioning and validation of 6-beam CHAMP fringe tracking } \\
\text { Take data using MIRC (4-T) with CHAMP }\end{array}$ \\
\hline 2011 Winter & $\begin{array}{l}\text { Design and build new 6-beam PC hardware } \\
\text { New camera readout for MIRC (needed for new xenomai OS) }\end{array}$ \\
\hline 2011 Spring & $\begin{array}{l}\text { Install new photometric channel hardware } \\
\text { Align fibers and MIRC combiner for 6 beams }\end{array}$ \\
\hline 2011 Summer & $\begin{array}{l}\text { On-sky tests of 6-telescope system, MIRC+CHAMP } \\
\text { Upgrade data pipeline }\end{array}$ \\
\hline
\end{tabular}

\subsection{Remaining Steps}

Table 1 shows the approximately timeline for the MIRC 6-telescope upgrade effort, including all the issues we have just discussed. Most of the remaining effort is devoted to software development, including some refinements to the realtime fringetracker algorithm and plenty of work on making the user interface friendly and appropriate for a general user CHARA "facility" instrument. The main researchers contributing to the upgrades are the postdoctoral fellows Fabien Baron (CHAMP realtime) and Stefan Kraus (CHAMP interfaces), UM PhD student Xiao Che (MIRC 6-T and PCs), research scientists Ettore Pedretti (Xenomai OS), Nathalie Thureau (GUIs), Rafael Millan-Gabet (auto alignment), CHARA deputy director Theo ten Brummelaar (CHARA integration), and Professor John Monnier (overall responsibility, data pipeline).

As an example of the user interfaces being developed, Figure 7 shows our current master control panel for the CHAMP fringe tracker. The design of this gui was based on the innerworkings of the CHAMP Fringe tracker and solicited advice from ESO VLTI instrument specialists who have experience the FINITO fringetracker on VLTI. When complete, this panel will allow easy control of fringe search strategy, fringe detection thresholds, and will offer realtime display of multiple performance metrics.

In addition to software work, we plan to take MIRC offline in Spring 2011 to do the final optical realignment for 6 fibers and to introduce the new photometric channel hardware. This will be the most critical period of the transition since the shift from 4 to 6 telescopes will become nominally irreversible (we can go back to 4 telescopes but not easily).

\section{FUTURE OF MIRC}

With the 6-beam upgrade and the sensitivity and calibration enhancements, we expect MIRC to remain scientifically productive for the next 5 years. It would be very beneficial to replace the MIRC detector (PICNIC) with a new detector of lower readnoise and to rebuild the MIRC front end to have a cryogenic spectrograph and a cold synthetic pupil to allow sensitive K-band observations. Another direction would be to bring imaging to visible wavelengths at CHARA by making a visible "MIRC" $\dagger$ The potential for fringe-tracking, imaging interferometry has just started to be explored and the next decade should be an interesting one.

\footnotetext{
${ }^{\dagger}$ M. Muterspaugh (Tennessee State University) was recently awarded an NSF grant to build a new visibile combiner for the NPOI Interferometer called VISION. VISION is currently planned to be based on the beam combination strategies of MIRC, i.e., an all-in-one, image-plane combiner using single-mode fibers.
} 


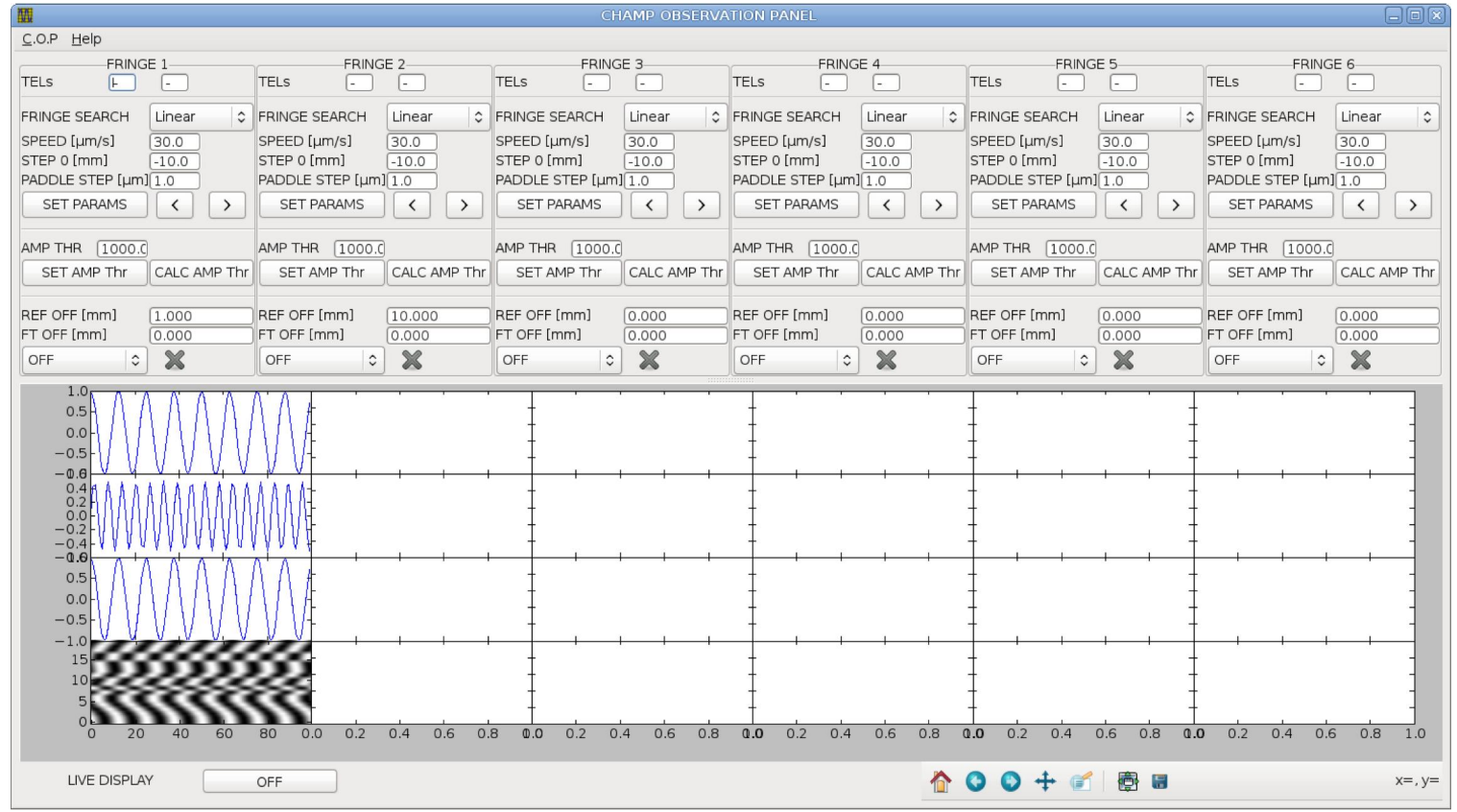

Figure 7. Here is a screenshot of our main control GUI for the CHAMP fringetracker. The interface was created using the python and its many publicly-available libraries.

\section{ACKNOWLEDGMENTS}

This work has been supported by the University of Michigan, the National Science Foundation (NSF-ST0352723,0707927,0807577) and NASA (NRA-NNG04GC00G,). Thanks also to UM machinist Scott Webster and the whole CHARA array team that makes science with MIRC possible.

\section{REFERENCES}

[1] Monnier, J. D., Berger, J.-P., Millan-Gabet, R., and Ten Brummelaar, T. A., "The Michigan Infrared Combiner (MIRC): IR imaging with the CHARA Array," in [New Frontiers in Stellar Interferometry, Proceedings of SPIE Volume 5491. Edited by Wesley A. Traub. Bellingham, WA: The International Society for Optical Engineering, 2004., p.1370], Traub, W. A., ed., Presented at the Society of Photo-Optical Instrumentation Engineers (SPIE) Conference 5491, 1370-+ (Oct. 2004).

[2] Monnier, J. D., Pedretti, E., Thureau, N., Berger, J.-P., Millan-Gabet, R., ten Brummelaar, T., McAlister, H., Sturmann, J., Sturmann, L., Muirhead, P., Tannirkulam, A., Webster, S., and Zhao, M., "Michigan Infrared Combiner (MIRC): commissioning results at the CHARA Array," in [Advances in Stellar Interferometry. Edited by Monnier, John D.; Schöller, Markus; Danchi, William C.. Proceedings of the SPIE, Volume 6268, pp. 62681P (2006).], Presented at the Society of Photo-Optical Instrumentation Engineers (SPIE) Conference 6268 (July 2006).

[3] ten Brummelaar, T. A., McAlister, H. A., Ridgway, S. T., Bagnuolo, Jr., W. G., Turner, N. H., Sturmann, L., Sturmann, J., Berger, D. H., Ogden, C. E., Cadman, R., Hartkopf, W. I., Hopper, C. H., and Shure, M. A., "First Results from the CHARA Array. II. A Description of the Instrument," ApJ 628, 453-465 (July 2005).

[4] Monnier, J. D., Zhao, M., Pedretti, E., Thureau, N., Ireland, M., Muirhead, P., Berger, J., Millan-Gabet, R., Van Belle, G., ten Brummelaar, T., McAlister, H., Ridgway, S., Turner, N., Sturmann, L., Sturmann, J., Berger, D., Tannirkulam, A., and Blum, J., "Imaging the surface of Altair and a MIRC update," in [Society of Photo-Optical Instrumentation Engineers (SPIE) Conference Series], Society of Photo-Optical Instrumentation Engineers (SPIE) Conference Series 7013 (July 2008). 
[5] Coudé du Foresto, V., Ridgway, S., and Mariotti, J., "Deriving object visibilities from interferograms obtained with a fiber stellar interferometer," A\&AS 121, 379-392 (Feb. 1997).

[6] Berger, D. H., Monnier, J. D., Millan-Gabet, R., ten Brummelaar, T. A., Muirhead, P., Pedretti, E., and Thureau, N., "CHARA Michigan phase-tracker (CHAMP)," in [Optical and Infrared Interferometry. Edited by Schöller, Markus; Proceedings of the SPIE, in press (2008).], Presented at the Society of Photo-Optical Instrumentation Engineers (SPIE) Conference (2008).

[7] Monnier, J. D., Zhao, M., Pedretti, E., Thureau, N., Ireland, M., Muirhead, P., Berger, J.-P., Millan-Gabet, R., Van Belle, G., ten Brummelaar, T., McAlister, H., Ridgway, S., Turner, N., Sturmann, L., Sturmann, J., and Berger, D., "Imaging the Surface of Altair," Science 317, 342- (July 2007).

[8] Zhao, M., Monnier, J. D., Pedretti, E., Thureau, N., Mérand, A., ten Brummelaar, T., McAlister, H., Ridgway, S. T., Turner, N., Sturmann, J., Sturmann, L., Goldfinger, P. J., and Farrington, C., "Imaging and Modeling Rapidly Rotating Stars: $\alpha$ Cephei and $\alpha$ Ophiuchi," ApJ 701, 209-224 (Aug. 2009).

[9] Zhao, M., Gies, D., Monnier, J. D., Thureau, N., Pedretti, E., Baron, F., Merand, A., ten Brummelaar, T., McAlister, H., Ridgway, S. T., Turner, N., Sturmann, J., Sturmann, L., Farrington, C., and Goldfinger, P. J., "First Resolved Images of the Eclipsing and Interacting Binary $\beta$ Lyrae," ApJ 684, L95-L98 (Sept. 2008).

[10] Kloppenborg, B., Stencel, R., Monnier, J. D., Schaefer, G., Zhao, M., Baron, F., McAlister, H., Ten Brummelaar, T., Che, X., Farrington, C., Pedretti, E., Sallave-Goldfinger, P. J., Sturmann, J., Sturmann, L., Thureau, N., Turner, N., and Carroll, S. M., "Infrared images of the transiting disk in the $\epsilon$ Aurigae system," Nature 464, 870-872 (Apr. 2010).

[11] Ireland, M. J., Monnier, J. D., and Thureau, N., "Monte-Carlo imaging for optical interferometry," in [Society of Photo-Optical Instrumentation Engineers (SPIE) Conference Series], Society of Photo-Optical Instrumentation Engineers (SPIE) Conference Series 6268 (July 2006).

[12] Pauls, T. A., Young, J. S., Cotton, W. D., and Monnier, J. D., "A Data Exchange Standard for Optical (Visible/IR) Interferometry," PASP 117, 1255-1262 (Nov. 2005).

[13] Baron, F. and Young, J. S., "Image reconstruction at Cambridge University," in [Society of Photo-Optical Instrumentation Engineers (SPIE) Conference Series], Society of Photo-Optical Instrumentation Engineers (SPIE) Conference Series $\mathbf{7 0 1 3}$ (July 2008).

[14] Buscher, D. F., "Direct maximum-entropy image reconstruction from the bispectrum," in [Very High Angular Resolution Imaging], J. G. Robertson \& W. J. Tango, ed., IAU Symposium 158, 91-+ (1994).

[15] Cotton, W., Monnier, J., Baron, F., Hofmann, K., Kraus, S., Weigelt, G., Rengaswamy, S., Thiébaut, E., Lawson, P., Jaffe, W., Hummel, C., Pauls, T., Schmitt, H., Tuthill, P., and Young, J., "2008 imaging beauty contest," in [Society of Photo-Optical Instrumentation Engineers (SPIE) Conference Series], Society of Photo-Optical Instrumentation Engineers (SPIE) Conference Series $\mathbf{7 0 1 3}$ (July 2008).

[16] Lawson, P. R., Cotton, W. D., Hummel, C. A., Baron, F., Young, J. S., Kraus, S., Hofmann, K., Weigelt, G. P., Ireland, M., Monnier, J. D., Thiébaut, E., Rengaswamy, S., and Chesneau, O., "2006 interferometry imaging beauty contest," in [Society of Photo-Optical Instrumentation Engineers (SPIE) Conference Series], Society of Photo-Optical Instrumentation Engineers (SPIE) Conference Series 6268 (July 2006).

[17] Lawson, P. R., Cotton, W. D., Hummel, C. A., Monnier, J. D., Zhao, M., Young, J. S., Thorsteinsson, H., Meimon, S. C., Mugnier, L. M., Le Besnerais, G., Thiebaut, E. M., and Tuthill, P. G., "An interferometry imaging beauty contest," in [Society of Photo-Optical Instrumentation Engineers (SPIE) Conference Series], W. A. Traub, ed., Society of Photo-Optical Instrumentation Engineers (SPIE) Conference Series 5491, 886+ (Oct. 2004).

[18] Zhao, M., Monnier, J. D., ten Brummelaar, T., Pedretti, E., and Thureau, N. D., "Exoplanet studies with CHARA-MIRC," in [Society of Photo-Optical Instrumentation Engineers (SPIE) Conference Series], Society of Photo-Optical Instrumentation Engineers (SPIE) Conference Series 7013 (July 2008).

[19] McAlister, H. A., ten Brummelaar, T. A., Gies, D. R., Huang, W., Bagnuolo, Jr., W. G., Shure, M. A., Sturmann, J., Sturmann, L., Turner, N. H., Taylor, S. F., Berger, D. H., Baines, E. K., Grundstrom, E., Ogden, C., Ridgway, S. T., and van Belle, G., "First Results from the CHARA Array. I. An Interferometric and Spectroscopic Study of the Fast Rotator $\alpha$ Leonis (Regulus)," ApJ 628, 439-452 (July 2005). 
[20] Tannirkulam, A., Monnier, J. D., Harries, T. J., Millan-Gabet, R., Zhu, Z., Pedretti, E., Ireland, M., Tuthill, P., ten Brummelaar, T., McAlister, H., Farrington, C., Goldfinger, P. J., Sturmann, J., Sturmann, L., and Turner, N., "A Tale of Two Herbig Ae Stars, MWC 275 and AB Aurigae: Comprehensive Models for Spectral Energy Distribution and Interferometry," ApJ 689, 513-531 (Dec. 2008).

[21] Benisty, M., Berger, J., Jocou, L., Labeye, P., Malbet, F., Perraut, K., and Kern, P., "An integrated optics beam combiner for the second generation VLTI instruments," A\&A 498, 601-613 (May 2009).

[22] Colavita, M. M., Wallace, J. K., Hines, B. E., Gursel, Y., Malbet, F., Palmer, D. L., Pan, X. P., Shao, M., Yu, J. W., Boden, A. F., Dumont, P. J., Gubler, J., Koresko, C. D., Kulkarni, S. R., Lane, B. F., Mobley, D. W., and van Belle, G. T., "The Palomar Testbed Interferometer," ApJ 510, 505-521 (Jan. 1999).

[23] Pedretti, E., Monnier, J. D., Thureau, N. D., and Berger, D. H., "A freely available real-time operating system well suited for astronomy and the physical sciences," in [Society of Photo-Optical Instrumentation Engineers (SPIE) Conference Series], Society of Photo-Optical Instrumentation Engineers (SPIE) Conference Series 6268 (July 2006). 